\title{
Another link in the chain
}

p53 - the guardian of the genome - is mutated in more than half of all human cancers, making it a prime therapeutic target. But p53 activity is regulated by many proteins; could any of these prove to be suitable candidates for cancer therapy? In the 19 October issue of Cell, Luo et al. and Vazri et al. identify another protein Sir 2 - that regulates p53's activity, and show that its inhibition potentiates $\mathrm{p} 53$ 's effects.

It has long been known that p53 activity is regulated by phosphorylation, but recent reports indicate that it is also regulated by a different type of modification - acetylation. Acetylation has previously been associated with the regulation of histones - the protein components of chromatin - but the Sir2 proteins are NADdependent deacetylases that are conserved in lower organisms that don't contain histones, so could they be involved in deacetylation of $\mathrm{p} 53$ ?

To investigate this, the groups isolated mammalian Sir2 homologues. Both human SIRT1 (also known as SIR $2 \alpha$ ) and mouse Sirt1 (also known as Sir $2 \alpha$ ) interacted with p53 in vitro and in vivo, and purified Sirt 1 could deacetylate p53 in vitro. The in vivo deacetylase activity of SIRT1 was confirmed using an antibody that was specific for acetylated p 53 . Acetylated $\mathrm{p} 53$ could not be detected following transfection of SIRT1.

So why is p53 acetylated? Acetylation seems to be induced in response to cellular stress. Luo et al. showed that $\mathrm{p} 53$ acetylation was induced following treatment with the DNA-damaging drug etoposide, and Vaziri et al. showed that there was an increase in p53 acetylation following $\gamma$-irradiation. Acetylation could be abolished by expression of SIRT1.

As p53 is acetylated in response to stress, it is logical to assume that this would correlate with an increase in its activity, and that deacetylation by SIRT 1 would inhibit p53's activity. The groups therefore tested the ability of $\mathrm{p} 53$ to activate transcription using a p53activated luciferase reporter system. Luo et al. generated a construct with p53-binding sites upstream of the transcription start site, and Vaziri et al. used the promoter of a 53 transcriptional target - the CDKN1A gene, which encodes WAF1 - to drive transcription. p53 increased luciferase activity in a dosedependent manner, and this activity was suppressed by SIRT1. Similar results were reported in vivo, as WAF1 was induced following exposure to $\gamma$-irradiation, and a fourfold overexpression of SIRT1 reduced this induction. So, SIRT1 inhibits p53's ability to activate transcription, but what about its ability to induce apoptosis? Luo et al. treated $\mathrm{p} 53^{+/+}$ cells with either etoposide or hydrogen peroxide (oxidative stress), both of which induce apoptosis. However, expression of Sirtl makes the cells more resistant to these types of cellular stress, so promoting cell survival. These results indicate that $\mathrm{p} 53$ acetylation enhances its activity, and that this is attenuated by SIRT1. So could inhibition of SIRT1 prove a useful therapeutic approach for restoring $\mathrm{p} 53$ activity in cancer cells?

The answer seems to be yes. Luo et al. exploited the knowledge that Sirt1 was dependent on NAD

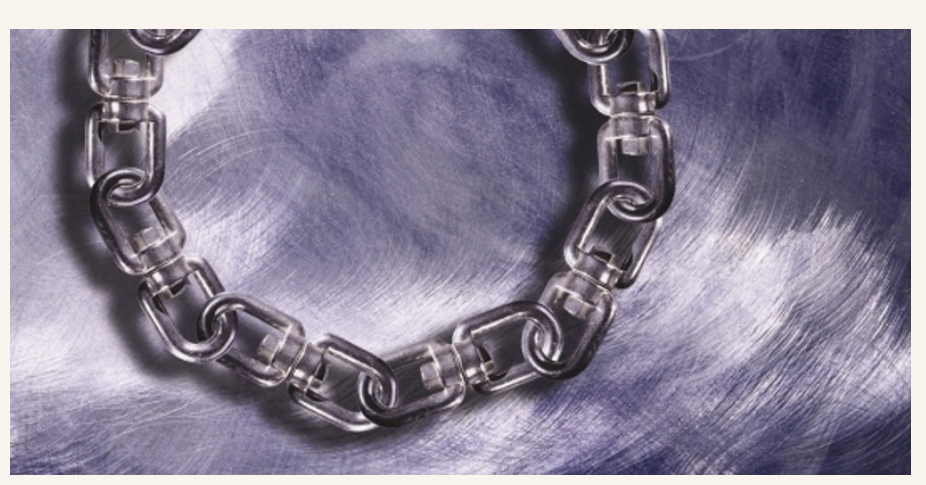

\section{WEB WATCH}

Chopping and changing

- http://www.infobiogen.fr/ services/chromcancer/

Can a single web site catalogue all the genetic changes in every type of cancer? This is the ambitious aim of the Atlas of Genetics and Cytogenetics in

Oncology and Haematology, although its editor, Jean-

Loup Huret (University Hospital, Poitiers, France), admits that the task will never be complete. The peerreviewed atlas allows users to search through several different headings. The 'genes' section contains concise summaries of oncogenes and tumour suppressors. Each gene has a 'card' listing its salient features, cancers in which the gene is implicated and links to other sources of information. The choice of entries belies a bias towards haematological malignancies, however, and there are some striking oversights (INK4A ARF and MDM2 are missing, for example).

The 'leukaemias' section shuffles the cards according to chromosomal rearrangement. Here, you'll find notes on clinical features, treatment, other cytogenetic abnormalities that cluster with the rearrangement in question, the genes involved and references. There's a similar section for solid tumours, this time organized according to tumour type. Other sections include a deck of cards on cancer-prone disorders, 'deep insight' articles, which go into more detail than is possible for the standard database entries, and links to related resources.

The database provides an enormous amount of information in a user-friendly format, but perhaps it would be more successful if it was less ambitious. For the cytogeneticist, it provides a useful adjunct to the Mitelman Database of Chromosome Aberrations in Cancer. The Atlas also welcomes contributions, so if your favourite gene or translocation is missing, why not let the curators know? 